\title{
UNIDADES DE MEDIDA
}

\section{MEASUREMENT UNITS}

\author{
Pedro RONZELLI JÚNIOR ${ }^{1}$
}

\section{RESUMO}

A importância e a necessidade de uniformização da utilização e apresentação das unidades nos trabalhos técnico-científicos foram as razões da realização dessa revisão de bibliografia. 0 objetivo foi o de contribuir para que haja aumento na qualidade dos manuscritos e aceitação cosmopolita das publicações. Foram analisadas e destacadas as normas sobre o Sistema Internacional de Unidades (SI), publicadas pelo Conselho Nacional de Metrologia, Normalização e Qualidade Industrial (CONMETRO), por meio de comentários sobre a grafia e apresentados quadros com as unidades de base, unidades suplementares, unidades derivadas, múltiplos e submúltiplos dessas unidades, unidades não pertencentes ao SI e toleradas e as mais utilizadas nas ciências agrárias.

Palavras-chave: Unidades, Sistema Internacional, Redação científica.

\begin{abstract}
The importance and necessity of uniformed units use and presentation in papers were the reasons to this bibliographical revision. The goal was to contribute to raise in quality the manuscripts and become cosmopolitan the acceptation of the publications. It was done an analysis about the rules of the International System of Units (SI) in Brazil, published by the Conselho Nacional de Metrologia, Normalização e Qualidade Industrial (CONMETRO), with comments about how to write correctly and also presented some tables with the base units, supplementary units, derived units, multiple and submultiples of these units, tolerated units out of the SI and some of the mostly used units in Agrarian sciences.
\end{abstract}

Key-words: Units, International System, Scientific text. 


\section{INTRODUÇÃO}

A redação científica é uma das formas pelas quais os pesquisadores se comunicam, assim, nada mais importante que a uniformização da linguagem para que todos, em qualquer lugar do planeta, possam ler e compreender o que se escreve. Considerando não só as necessidades de comunicação equânime entre pesquisadores, mas também a de outros segmentos, foram tomadas no Brasil, há alguns anos, providências de âmbito geral na direção de normalizar a utilização de unidades como um caminho natural a ser seguido.

O Conselho Nacional de Metrologia, Normalização e Qualidade Industrial (CONMETRO) é o organismo brasileiro responsável por, entre outras atribuições, realizar a normalização da utilização de unidades. Assim, o CONMETRO, usando das atribuições que lhe confere 0 artigo $3^{\circ}$ da Lei $n^{\circ}$ 5.966, de 11 de dezembro de 1973, na 20 a Sessão Ordinária realizada em Brasília, em 23 de outubro de 1988, considerando que as unidades de medida legais no Brasil são aquelas do Sistema Internacional de Unidades (SI) [Système International d'Unités], adotado pela Conferência Geral de Pesos e Medidas (CGPM), cuja adesão pelo Brasil foi formalizada mediante o Decreto Legislativo no 57, de 27 de junho de 1953, e considerando a necessidade de assegurar, em todo o Território Nacional, a indispensável uniformidade na expressão quantitativa e metrológica das grandezas de quaisquer atividades comerciais, agropecuárias, industriais, técnicas ou científicas, decidiu, pela Resolução № 12/1988: a) adotar o Quadro Geral de Unidades de Medida (QGU), que é apresentado pelos Quadros 1 a 9, nos quais constam nomes, definições, símbolos e prefixos das unidades; b) aceitar o emprego de algumas unidades fora do $\mathrm{SI}$, quando indispensáveis para determinadas medições; e c) estabelecer o Instituto Nacional de Metrologia, Normalização e Qualidade Industrial (INMETRO) como encarregado de propor modificações, quando necessárias, e resolver os casos omissos na aplicação das unidades legais.

\section{O SISTEMA INTERNACIONAL DE UNIDADES (SI)}

O SI é composto por sete unidades de base, duas unidades suplementares, algumas unidades derivadas e múltiplos e submúltiplos decimais dessas unidades. São também aceitos, no QGU, dois tipos de unidades fora do SI, aquelas aceitas para utilização com o SI, isoladamente ou combinadas entre si e/ou com unidades SI, sem restrição de prazo e outras admitidas temporariamente.
O SI foi adotado pela $11^{\text {a }}$ CGPM, realizada em 1960, e a partir dessa adoção algumas entidades internacionais como a União Internacional de Química Pura e Aplicada (IUPAC), a União Internacional de Física Pura e Aplicada (IUPAP), a Organização Internacional para Padronização (ISO) e o Bureau Internacional de Pesos e Medidas (BIPM) têm regulamentado a sua utilização. $\mathrm{Na}$ mesma linha da uniformização da linguagem e de conceitos, algumas editoras e periódicos das mais variadas áreas da ciência, inclusive das ciências agrárias, estão adotando o SI.

\section{GRAFIA DAS UNIDADES E DOS NÚMEROS}

Os símbolos das unidades devem ser expressos por letras minúsculas, exceto quando derivados de nomes próprios. Quando os nomes forem escritos por extenso começam por letra minúscula, mesmo quando têm o nome de um cientista como, por exemplo, ampère, kelvin, newton e outros, exceto 0 grau Celsius.

A grafia das unidades, por extenso, é comum, porém, deve haver cuidados com o plural. Nesses casos, os prefixos são invariáveis. Os nomes de unidades, quando palavras simples, recebem um "s" no final como, por exemplo, ampéres, curies, candelas, farads, grays, joules, kelvins, volts etc. Quando palavras compostas, sem hífen, o plural é feito flexionando as duas ou mais palavras como, por exemplo, metros quadrados, milhas marítimas etc. Quando termos compostos, por força da multiplicação que representam, variam independentemente e recebem um hífen por complemento como, por exemplo, newtons-metros, pascals-segundos etc. Deve-se ter cuidados com a não observância dos plurais regulares nos casos de becquerels, decibels, henrys, mols, pascals etc. Alguns nomes de unidades não recebem a letra "s" no final quando terminam em s, x ou z como, por exemplo, siemens, lux, hertz etc. Também não recebem o "s" quando representam o denominador em unidades compostas por divisão como, por exemplo, quilômetros por hora, lumens por watt etc., e quando em palavras compostas, em que são complementares de nomes de outras unidades e ligadas por hífen ou preposição como, por exemplo, anos-luz, quilogramas-força etc.

Os símbolos devem ser grafados sem complementos como a letra "s" para indicar plural ou o ponto para indicar a abreviatura. Utilizando como exemplo a unidade de medida metro, cujo símbolo é a letra eme minúscula $(\mathrm{m})$, observa-se que a mesma não deve ser seguida de qualquer sinal gráfico, de tal modo que, tanto para indicar um metro $(1,0 \mathrm{~m})$ quanto para indicar dez metros $(10,0 \mathrm{~m})$, o símbolo permanece inalterado. Observe-se que a flexão 
acontece apenas na redação por extenso. Todos os símbolos devem ser grafados no mesmo plano ou alinhamento do número a que se refere e não como seu expoente. São exceções os símbolos das unidades não pertencentes ao SI referentes a ângulo

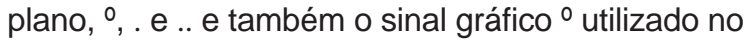
símbolo do grau Celsius.

Os volumes devem ser expressos em metros cúbicos $\left(\mathrm{m}^{3}\right)$ e seus múltiplos. Essa é a recomendação, porém, o litro (L ou I) e seus múltiplos são também aceitáveis. O símbolo do litro pode ser escrito tanto com letra minúscula quanto maiúscula. Esta última é preferida por evitar confusão com o algarismo um (1) em alguns tipos de fontes, como é o caso da "Times New Roman".

A unidade de base de massa é o quilograma $(\mathrm{kg})$. Seus múltiplos e submúltiplos devem ser formados com os prefixos do Quadro 1 e a palavra grama. Deve haver atenção para o termo tonelada (t), equivalente a $1.000 \mathrm{~kg}$, que pode ser e é empregado no lugar de megagrama $(\mathrm{Mg})$, principalmente por essa grafia ser idêntica à do elemento Magnésio. A unidade megagrama tem sido largamente utilizada em publicações na língua inglesa, uma vez que a tonelada inglesa é diferente da tonelada métrica. Assim, nos trabalhos publicados em inglês, talvez seja preferível empregar megagrama no lugar de tonelada.

A unidade SI para tempo é o segundo (s), mas o uso de minuto (min), hora (h) e dia (d) é aceitável em muitos casos e até preferível a unidades como o decassegundo (das) ou o megassegundo (Ms). Deve-se evitar o uso do mês como unidade em razão de seu valor ser variável de 28 a 31 dias.

A unidade SI para temperatura é o grau kelvin $(\mathrm{K})$, mas o grau Celsius $\left({ }^{\circ} \mathrm{C}\right)$ também é aceitável e é o mais freqüentemente utilizado. A conversão de um para outro é feita utilizando a fórmula: ${ }^{\circ} \mathbf{C}=\mathrm{K}-\mathbf{2 7 3}, \mathbf{1 5}$. O termo "grau centígrado" é tido como obsoleto, encontrando-se em desuso, e deve ser substituído por "grau Celsius".

A unidade SI para matéria é o mol. O mol é definido como a quantidade de matéria de um sistema que contém tantas unidades elementares quantos forem os átomos contidos em $0,012 \mathrm{~kg}$ de Carbono12. Como conseqüência da definição de mol, as expressões equivalente grama (e), normal (N) e normalidade tornaram-se obsoletas e estão em desuso.

A utilização da percentagem (\%) deve acontecer apenas nos casos em que for estritamente necessária. Por outro lado, unidades como partes por mil, partes por milhão (ppm) e partes por bilhão (ppb) devem ter sua utilização evitada e até eliminada, uma vez que essas unidades não fazem parte do SI. O mesmo deve ser feito com unidades como atmosfera, libra por polegada quadrada (psi), mm de Hg e bar, que devem ser substituídas, em razão de a unidade SI de pressão ser o pascal (Pa), que é a pressão exercida por uma força de $1 \mathrm{~N} . \mathrm{m}^{-2}$. Para medidas de radioatividade, o curiel deve ser substituído pelo becquerel (Bq).

Os números, exceto aqueles que não representam quantidades, por exemplo, numeração de elementos em seqüência, códigos de identificação, datas, números de telefones etc., deverão sempre ter a parte inteira separada da parte decimal por uma vírgula. Quando representam quantias em dinheiro ou quantidades de mercadorias ou serviços em documentos para efeitos fiscais, jurídicos e/ou comerciais, devem ser grafados com os algarismos separados por um ponto, em grupos de três, tanto à direita quanto à esquerda da vírgula, por exemplo, $10.000,00$ ou $0,234.759$. Nos demais casos, principalmente o dos trabalhos técnicocientíficos, existe a recomendação da separação dos algarismos em grupos de três, não necessariamente por acréscimo de ponto ou outro sinal gráfico como, por exemplo, 114057 árvores ou 114.057 árvores.

O espaçamento entre o número e o símbolo da unidade deve atender à conveniência de cada caso. No texto corrente dos trabalhos técnicocientíficos, deve-se dar o espaço. A dispensa desse espaço somente se aplica quando há possibilidade de fraude. No caso de quadros, é facultada a utilização do espaço. Para esses trabalhos também se determina a utilização dos prefixos SI ou fatores decimais apresentados no Quadro 1.

\section{AS UNIDADES}

O SI ratificado pela 11ㄹ CGPM, de 1960, e atualizado pela 18 ${ }^{\mathrm{a}} \mathrm{CGPM}$, de 1987, é composto por sete unidades de base ou fundamentais e duas suplementares. Essas unidades são consideradas, por convenção, como dimensionalmente independentes e são apresentadas no Quadro 2.

As unidades derivadas são expressões algébricas das unidades de base e são apresentadas no Quadro 3. Algumas dessas unidades recebem nomes e símbolos especiais, apresentados no Quadro 4, e podem ser utilizadas para definir outras grandezas derivadas, apresentadas no Quadro 5. Há ainda as unidades SI derivadas formadas a partir das unidades suplementares, que estão no Quadro 6.

A utilização de unidades não pertencentes ao SI deve ser restrita, a fim de preservar as vantagens de um sistema homogêneo. No entanto, algumas unidades são necessárias e convenientes, como é o caso das apresentadas no Quadro 7. Nos Quadros 8 e 9 são listadas e apresentadas algumas unidades mais comuns nos trabalhos técnico-científicos das ciências agrárias e no Quadro 10 os fatores de conversão de unidades freqüentemente utilizadas e que não pertencem ao SI. 
QUADRO 1 - Prefixos do sistema internacional $(\mathrm{SI})^{(1)}$

\begin{tabular}{|c|c|c|c|}
\hline \multicolumn{2}{|c|}{ NOME DO PREFIXO } & \multirow{2}{*}{$\begin{array}{c}\text { SÍMBOLO DO } \\
\text { PREFIXO }\end{array}$} & \multirow{2}{*}{$\begin{array}{c}\text { FATOR DE } \\
\text { MULTIPLICAÇÃO }\end{array}$} \\
\hline BRASIL & EUA & & \\
\hline (2) & yocto & $y$ & $10^{-24}$ \\
\hline (2) & zepto & $z$ & $10^{-21}$ \\
\hline atto & atto & a & $10^{-18}$ \\
\hline femto & femto & $f$ & $10^{-15}$ \\
\hline pico & ouci & $p$ & $10^{-12}$ \\
\hline nano & nano & $\mathrm{n}$ & $10^{-9}$ \\
\hline micro & micro & $\mu$ & $10^{-6}$ \\
\hline mili & nukku & $\mathrm{m}$ & $10^{-3}$ \\
\hline centi & centi & c & $10^{-2}$ \\
\hline deci & deci & d & $10^{-1}$ \\
\hline deca & deca & da & $10^{1}$ \\
\hline hecto & hecto & $\mathrm{h}$ & $10^{2}$ \\
\hline quilo & kilo & $\mathrm{k}$ & $10^{3}$ \\
\hline mega & mega & M & $10^{6}$ \\
\hline giga & giga & G & $10^{9}$ \\
\hline tera & tera & $T$ & $10^{12}$ \\
\hline oeta & peta & $P$ & $10^{15}$ \\
\hline exa & exa & E & $10^{18}$ \\
\hline (2) & zetta & z & $10^{21}$ \\
\hline (2) & yotta & $\mathrm{Y}$ & $10^{24}$ \\
\hline
\end{tabular}

Dados rearranjados pelo autor, fonte: CANTARELLA e ANDRADE (1992).

2 Prefixos ainda não regulamentados no Brasil.

QUADRO 2 - Unidades de base e suplementares do sistema internacional (SI) ${ }^{(1)}$

\begin{tabular}{lcc}
\hline \multicolumn{1}{c}{ GRANDEZA } & UNIDADE & SímBOLO \\
\hline Comprimento & UNIDADES DE BASE & \\
Massa & metro & $\mathrm{m}$ \\
Tempo & quilograma & $\mathrm{kg}$ \\
Corrente elétrica & segundo & $\mathrm{s}$ \\
Temperatura termodinâmica & ampère & $\mathrm{A}$ \\
Quantidade de matéria & kelvin & $\mathrm{K}$ \\
Intensidade luminosa & mol & $\mathrm{mol}$ \\
& candela & $\mathrm{cd}$ \\
Ângulo plano & UNIDADES SUPLEMENTARES & \\
Ângulo sólido & radiano & $\mathrm{rad}$ \\
\hline
\end{tabular}

1 Fonte: CANTARELLA e ANDRADE (1992). 
QUADRO 3 - Algumas unidades do sistema internacional (SI) derivadas simples, expressas em termos de unidades básicas ${ }^{(1)}$

\begin{tabular}{lll}
\hline \multicolumn{1}{c}{ GRANDEZA } & \multicolumn{1}{c}{ UNIDADE } & SíMBOLO \\
\hline Área & metro quadrado & $\mathrm{m}^{2}$ \\
Volume & metro cúbico & $\mathrm{m}^{3}$ \\
Velocidade & metro por segundo & $\mathrm{m}^{-1} \mathrm{~s}^{-2}$ \\
Aceleração & metro por segundo ao quadrado & $\mathrm{m} \cdot \mathrm{s}^{-2}$ \\
Densidade & quilograma por metro cúbico & $\mathrm{kg} \cdot \mathrm{m}^{-3}$ \\
Concentração em quantidade de matéria & mol por metro cúbico & $\mathrm{mol}^{-3}$ \\
Densidade de corrente & ampère por metro quadrado & $\mathrm{A} \cdot \mathrm{m}^{-2}$ \\
Força de campo magnético & ampère por metro & $\mathrm{A} \cdot \mathrm{m}^{-1}$ \\
Volume específico & metro cúbico por quilograma & $\mathrm{m}^{3} \cdot \mathrm{kg}^{-1}$ \\
Luminância & candela por metro quadrado & $\mathrm{cd} \cdot \mathrm{m}^{-2}$ \\
\hline
\end{tabular}

1 Fonte: CANTARELLA e ANDRADE (1992).

QUADRO 4 - Algumas unidades do sistema internacional (SI) derivadas com nomes e símbolos especiais(1)

\begin{tabular}{|c|c|c|c|}
\hline \multirow[b]{2}{*}{ GRANDEZA } & \multicolumn{3}{|c|}{ UNIDADE SI } \\
\hline & NOME & SÍMBOLO & DEFINIÇÃO \\
\hline Força & newton & $\mathrm{N}$ & $\mathrm{m} \cdot \mathrm{kg} \cdot \mathrm{s}^{-2}$ \\
\hline Pressão & pascal & $\mathrm{Pa}$ & $\mathrm{m}^{-1} \cdot \mathrm{kg} \cdot \mathrm{s}^{-2}\left(=\mathrm{N} \cdot \mathrm{m}^{-2}\right)$ \\
\hline Energia, trabalho, quantidade de calor & joule & $\mathrm{J}$ & $\mathrm{m}^{2} \cdot \mathrm{kg} \cdot \mathrm{s}^{-2}(=\mathrm{N} \cdot \mathrm{m})$ \\
\hline Potência, fluxo radiante & watt & W & $\mathrm{m}^{2} \cdot \mathrm{kg} \cdot \mathrm{s}^{-3}\left(=\mathrm{J} \cdot \mathrm{s}^{-1}\right)$ \\
\hline Carga elétrica, quantidade de eletricidade & coulomb & C & S.A \\
\hline Diferença de potencial, potencial elétrico & volt & $\mathrm{V}$ & $m^{2} \cdot k g \cdot s^{-3} \cdot A^{-1}\left(=W \cdot A^{-1}\right)$ \\
\hline Resistência elétrica & ohm & $\Omega$ & $\mathrm{m}^{2} \cdot \mathrm{kg} \cdot \mathrm{s}^{-3} \cdot \mathrm{A}^{-2}\left(=V \cdot \mathrm{A}^{-1}\right)$ \\
\hline Condutância elétrica & siemens & S & $\mathrm{m}^{-2} \cdot \mathrm{kg}^{-1} \cdot \mathrm{s}^{3} \cdot \mathrm{A}^{2}\left(=\Omega^{-1}=\mathrm{A} \cdot \mathrm{V}^{1}\right)$ \\
\hline Capacitância elétrica & farad & $\mathrm{F}$ & $m^{-2} \cdot \mathrm{kg}^{-1} \cdot \mathrm{s}^{4} \cdot \mathrm{A}^{2}\left(=\mathrm{C} \cdot \mathrm{V}^{-1}\right)$ \\
\hline Fluxo magnético & weber & $\mathrm{Wb}$ & $\mathrm{m}^{2} \cdot \mathrm{kg} \cdot \mathrm{s}^{-2} \cdot \mathrm{A}^{-1}(=\mathrm{V} \cdot \mathrm{s})$ \\
\hline Indutância & henry & $\mathrm{H}$ & $\mathrm{m}^{2} \cdot \mathrm{kg} \cdot \mathrm{s}^{-2} \cdot \mathrm{A}^{-2}\left(=\mathrm{Wb} \cdot \mathrm{A}^{-1}\right)$ \\
\hline Indução magnética & tesla & $\mathrm{T}$ & $\mathrm{kg} \cdot \mathrm{s}^{-2} \cdot \mathrm{A}^{-1}\left(=\mathrm{Wb} \cdot \mathrm{m}^{-2}\right)$ \\
\hline Freqüência & hertz & $\mathrm{Hz}$ & $s^{-1}$ \\
\hline Fluxo luminoso & lúmen & Im & cd.sr ${ }^{(2)}$ \\
\hline lluminância & lux & lx & $\mathrm{m}^{-2} \cdot \mathrm{cd} \cdot \mathrm{sr}{ }^{(2)}\left(=\mathrm{Im} \cdot \mathrm{m}^{-2}\right)$ \\
\hline Atividade de um radionuclídeo & becquerel & $\mathrm{Bq}$ & $s^{-1}$ \\
\hline Dose absorvida & gray & Gy & $\mathrm{m}^{2} \cdot \mathrm{s}^{-2}\left(=\mathrm{j} \cdot \mathrm{kg}^{-1}\right)$ \\
\hline Dose equivalente & sievert & Sv & $\mathrm{m}^{2} \cdot \mathrm{s}^{-2}\left(=\mathrm{j} \cdot \mathrm{kg}^{-1}\right)$ \\
\hline Temperatura Celsius & grau Celsius & ${ }^{\circ} \mathrm{C}$ & $\mathrm{K}$ \\
\hline
\end{tabular}

1 Fonte: CANTARELLA e ANDRADE (1992).

2 Esterradiano (unidade suplementar, nesta expressão tratada como unidade de base).

QUADRO 5 - Algumas unidades do sistema internacional (SI) derivadas, expressas em termos de unidades com nomes especiais ${ }^{(1)}$

\begin{tabular}{|c|c|c|c|}
\hline GRANDEZA & UNIDADE & SÍMBOLO & DEFINIÇĀO \\
\hline $\begin{array}{l}\text { Viscosidade dinâmica } \\
\text { Momento de força } \\
\text { Tensão superficial } \\
\text { Densidade de potência, irradiância } \\
\text { Entropia } \\
\text { Entropia específica } \\
\text { Energia específica } \\
\text { Condutividade térmica } \\
\text { Densidade de energia } \\
\text { Força de campo elétrico } \\
\text { Densidade de carga elétrica } \\
\text { Densidade de fluxo elétrico } \\
\text { Permitividade } \\
\text { Energia molar } \\
\text { Entropia molar } \\
\text { Exposição (Raios X e gama) } \\
\text { Taxa de dose absorvida } \\
\end{array}$ & $\begin{array}{l}\text { pascal-segundo } \\
\text { newton-metro } \\
\text { newton por metro } \\
\text { watt por metro quadrado } \\
\text { joule por kelvin } \\
\text { joule por kelvin-quilograma } \\
\text { joule por quilograma } \\
\text { watt por metro-kelvin } \\
\text { joule por metro cúbico } \\
\text { volt por metro } \\
\text { coulomb por metro cúbico } \\
\text { coulomb por metro quadrado } \\
\text { farad por metro } \\
\text { joule por mol } \\
\text { joule por kelvin-mol } \\
\text { coulomb por quilograma } \\
\text { gray por segundo }\end{array}$ & 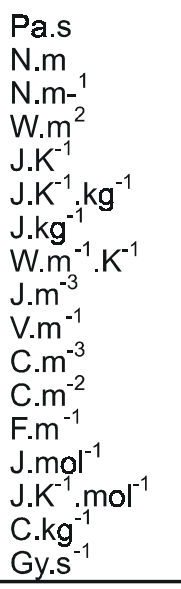 & $\begin{array}{c}\mathrm{m}^{-1} \cdot \mathrm{kg} \cdot \mathrm{s}^{-1} \\
\mathrm{~m}^{2} \cdot \mathrm{kg} \cdot \mathrm{s}^{-2} \\
\mathrm{~kg} \cdot \mathrm{s}^{-2} \\
\mathrm{~kg} \cdot \mathrm{s}^{-3} \\
\mathrm{~m}^{2} \cdot \mathrm{kg} \cdot \mathrm{s}^{-2} \cdot \mathrm{K}^{-1} \\
\mathrm{~m}^{2} \cdot \mathrm{s}^{-2} \cdot \mathrm{K}^{-1} \\
\mathrm{~m}^{2} \cdot \mathrm{s}^{-2} \\
\mathrm{~m} \cdot \mathrm{kg} \cdot \mathrm{s}^{-3} \cdot \mathrm{K}^{-1} \\
\mathrm{~m}^{-1} \cdot \mathrm{kg}^{-2} \mathrm{~s}^{-2} \\
\mathrm{~m}^{\mathrm{kgg}} \cdot \mathrm{s}^{-3} \cdot \mathrm{A}^{-1} \\
\mathrm{~m}^{-3} \cdot \mathrm{s} \cdot \mathrm{A} \\
\mathrm{m}^{-2} \cdot \mathrm{s} \cdot \mathrm{A} \\
\mathrm{m}^{-3} \cdot \mathrm{kg} \mathrm{g}^{-1} \cdot \mathrm{s}^{4} \cdot \mathrm{A}^{2} \\
\mathrm{~m}^{2} \cdot \mathrm{kg}^{-2} \cdot \mathrm{s}^{-2} \cdot \mathrm{mol}^{-1} \\
\mathrm{~m}^{2} \cdot \mathrm{kg}^{-2} \cdot \mathrm{s}^{-2} \cdot \mathrm{K}^{-1} \cdot \mathrm{mol}^{-1} \\
\mathrm{~kg}{ }^{-1} \cdot \mathrm{s} \cdot \mathrm{A} \\
\mathrm{m}^{2} \cdot \mathrm{s}^{-3}\end{array}$ \\
\hline
\end{tabular}

1 Fonte: CANTARELLA e ANDRADE (1992). 
QUADRO 6 - Unidades do sistema internacional (SI) derivadas, formadas usando-se unidades SI suplementares ${ }^{(1)}$

\begin{tabular}{lll}
\hline \multicolumn{1}{c}{ GRANDEZA } & \multicolumn{1}{c}{ UNIDADE } & SÍMBOLO \\
\hline Velocidade angular & radiano por segundo & ${\mathrm{rad} . \mathrm{s}^{-1}}^{-2}$ \\
Aceleração angular & radiano por segundo quadrado & $\mathrm{rad} . \mathrm{s}^{-2}$ \\
Intensidade energética & watt por esterradiano & $\mathrm{W} . \mathrm{sr}^{-1}$ \\
Luminância energética & watt por esterradiano-metro quadrado & $\mathrm{W} . \mathrm{sr}^{-1} \cdot \mathrm{m}^{-2}$ \\
\hline
\end{tabular}

1 Fonte: CANTARELLA e ANDRADE (1992)

QUADRO 7 - Unidades não pertencentes ao sistema internacional (SI), normalmente aceitas para uso com unidades $\mathrm{SI}^{(1)}$

\begin{tabular}{clll}
\hline GRANDEZA & UNIDADE & SÍMBOLO & TRANSPOSIÇÃO PARA UNIDADE SI \\
\hline \multirow{2}{*}{ Tempo } & minuto & min & $1 \mathrm{~min}=60 \mathrm{~s}$ \\
& hora & $\mathrm{h}$ & $1 \mathrm{~h}=60 \mathrm{~min}=3.600 \mathrm{~s}$ \\
Ângulo plano & dia & $\mathrm{d}$ & $1 \mathrm{~d}=24 \mathrm{~h}=86.400 \mathrm{~s}$ \\
& grau & $\circ$ & $1^{\circ}=(\pi / 180) \mathrm{rad}$ \\
Volume & minuto & ' & $1^{\prime}=(1 / 60)^{\circ}=(\pi / 10.800) \mathrm{rad}$ \\
Massa & segundo & " & $1^{\prime \prime}=(1 / 60)^{\prime}=(\pi / 648.000) \mathrm{rad}$ \\
Área & litro & I ou L & $1 \mathrm{~L}=1 \mathrm{dm}^{3}=10^{-3} \mathrm{~m}^{3}$ \\
\hline
\end{tabular}

1 Fonte: CANTARELLA e ANDRADE (1992)

QUADRO 8 - Alguns exemplos de unidades mais comuns em trabalhos técnico científicos das ciências agrárias ${ }^{(1)}$

\begin{tabular}{|c|c|c|}
\hline QUANTIDADE/TAXA & APLICAÇÃO & UNIDADE/SÍMBOLO \\
\hline $\begin{array}{l}\text { Ângulo } \\
\text { Área }\end{array}$ & $\begin{array}{l}\text { Difração de raio } X \\
\text { Área de terreno } \\
\text { Área de vaso } \\
\text { Área foliar } \\
\text { Área superficial específica (solo) } \\
\text { Razão de área foliar }\end{array}$ & $\begin{array}{l}\text { radiano }(\theta) \text {, grau }\left(^{\circ}\right) \\
\text { ha } \\
\mathrm{cm}^{2} \\
\mathrm{~m}^{2} \\
\mathrm{~m}^{2} \cdot \mathrm{kg}^{-1} \\
\mathrm{~m}^{2} \cdot \mathrm{kg}^{-1} \text { de planta }\end{array}$ \\
\hline Volume & $\begin{array}{l}\text { Vaso } \\
\text { Tanques, campo } \\
\text { Frascos }\end{array}$ & $\begin{array}{l}\mathrm{dm}^{3}, \mathrm{~L} \\
\mathrm{~m}^{3} \\
\mathrm{~cm}^{3}, \mathrm{~mL}\end{array}$ \\
\hline Comprimento & $\begin{array}{l}\text { Profundidade de solo } \\
\text { Espaçamento } \\
\text { Espaço interatômico }\end{array}$ & $\begin{array}{l}\mathrm{m}, \mathrm{cm} \\
\mathrm{m} \\
\mathrm{nm}\end{array}$ \\
\hline Temperatura & Solo, ar etc. & ${ }^{\circ} \mathrm{C}, \mathrm{K}$ \\
\hline Concentração & $\begin{array}{l}\text { Massa molar conhecida } \\
\text { - meio líquido } \\
\text { - solo, absorção de íons } \\
\text { - nutriente em plantas } \\
\text { Massa molar desconhecida }\end{array}$ & $\begin{array}{l}\text { mol.m } \mathrm{m}^{-3}, \mathrm{~mol} \cdot \mathrm{kg}, \mathrm{g}, \mathrm{kg} \mathrm{g}^{-1}, \mathrm{~g} \cdot \mathrm{L}^{-1}(2) \\
\mathrm{mol} \cdot \mathrm{m}^{-3}, \mathrm{cmol} \cdot \mathrm{kg}^{-1}, \mathrm{~g} \cdot \mathrm{kg}^{-1}, \mathrm{~g} \cdot \mathrm{dm}^{-1(2)} \\
\mathrm{mmol} \cdot \mathrm{kg}^{-1}, \mathrm{~g} \cdot \mathrm{kg}^{-1(2)}\end{array}$ \\
\hline & $\begin{array}{l}\text { - meio líquido } \\
\text { - solo, absorção de ions } \\
\text { - nutriente em plantas } \\
\text { Conteúdo de água na planta } \\
\text { Conteúdo de água no solo } \\
\text { Gases } \\
\text { Fertilizantes }\end{array}$ & $\begin{array}{l}\mathrm{kg} \cdot \mathrm{m}^{-3}, \mathrm{~g} \cdot \mathrm{L}^{-1} \\
\mathrm{~kg} \cdot \mathrm{m}^{-3}, \mathrm{~g} \cdot \mathrm{L}^{-1}, \mathrm{~g} \cdot \mathrm{kg}^{-1}, \mathrm{mg} \cdot \mathrm{kg}^{-1}, \mathrm{mg} \cdot \mathrm{dm}^{-3}, \\
\mathrm{~g} \cdot \mathrm{kg}^{-1}, \mathrm{mg} \cdot \mathrm{kg}^{-1} \\
\mathrm{~g} \cdot \mathrm{kg}^{-1}, \mathrm{~kg} \cdot \mathrm{kg}^{-1} \\
\mathrm{~kg} \cdot \mathrm{kg}^{-1}, \mathrm{~m}^{3} \cdot \mathrm{m}^{-3} \\
\mathrm{~mol} \cdot \mathrm{m}^{-3}, \mathrm{~kg} \cdot \mathrm{m}^{-3} \mathrm{~g} \cdot \mathrm{L}^{1}, \mathrm{~mL} \cdot .^{1} \mathrm{~mol} \cdot \mathrm{mol}^{-1} \\
\mathrm{~g} \cdot \mathrm{m}^{-2}, \mathrm{kf} \cdot \mathrm{ha}^{-1}\end{array}$ \\
\hline Produtividade, Produção & $\begin{array}{l}\text { Grãos, matéria seca } \\
\text { Parte da planta }\end{array}$ & $\begin{array}{l}\text { t.ha }{ }^{-1}, \mathrm{Mg} \cdot \mathrm{ha}^{-1}, \mathrm{~kg} \mathrm{ha}^{-1}, \mathrm{~g} \cdot \mathrm{m}^{-2} \\
\text { g.planta } \\
\text { g.espiga }\end{array}$ \\
\hline Capacidade de troca & Solo & $\begin{array}{ll}\mathrm{mol}\left(\text { (́on). } \mathrm{kg}^{-1},\right. & \mathrm{cmol}\left(\text { carga) } . \mathrm{kg}^{-1},\right. \\
\mathrm{mol}\left(\text { carga) } \mathrm{kg}^{-1}\right. & \end{array}$ \\
\hline $\begin{array}{l}\text { Transporte de íons } \\
\text { Densidade aparente }\end{array}$ & $\begin{array}{l}\text { Absorção de ions } \\
\text { Solo }\end{array}$ & $\begin{array}{l}\text { mol(ion). } \mathrm{kgg}^{-1} \mathrm{~s}^{-1}, \mathrm{~mol} \text { (carga). } \mathrm{kg} \mathrm{s}^{-1} \\
\mathrm{~g} \cdot \mathrm{cm}^{-3}, \mathrm{~kg} \cdot \mathrm{dm}^{-3}, \mathrm{Mg}^{-3} \mathrm{~m}^{-3}\end{array}$ \\
\hline
\end{tabular}


continuação

\begin{tabular}{|c|c|c|}
\hline QUANTIDADE/TAXA & APLICAÇÃO & UNIDADE/SÍMBOLO \\
\hline Textura do solo & Solo & g. $\mathrm{kg}^{-1}, \%$ \\
\hline Precipitação & Chuva & $\mathrm{mm}$ \\
\hline Taxa de elongação & Planta & $m m \cdot s^{-1}, m m \cdot d^{-1}, m \cdot d^{-1}$ \\
\hline Taxa de crescimento & Planta & $g \cdot m^{-2} \cdot d^{-1}$ \\
\hline $\begin{array}{l}\text { Taxa } \\
\text { evapotranspiração }\end{array}$ & Solo e planta & $\mathrm{nm} \cdot \mathrm{s}^{-1}, \mathrm{~mm} \cdot \mathrm{d}^{-1}$ \\
\hline $\begin{array}{l}\text { Taxa de transpiração } \\
\text { foliar }\end{array}$ & Densidade de fluxo de água & $\mathrm{g} \cdot \mathrm{m}^{-2} \cdot \mathrm{s}^{-1}, \mathrm{~m}^{3} \cdot \mathrm{m}^{-2} \cdot \mathrm{s}^{-1}, \mathrm{~m} \cdot \mathrm{s}^{-1}$ \\
\hline Taxa de fotossíntese & $\begin{array}{l}\text { Densidade de fluxo } \\
\text { - quantidade de substância } \\
\text { - massa de } \mathrm{CO}_{2}\end{array}$ & $\begin{array}{l}\mu \mathrm{mol} \cdot \mathrm{m}^{-2} \cdot \mathrm{s}^{-1} \\
\mathrm{mg} \cdot \mathrm{m}^{-2} \cdot \mathrm{s}^{-1}\end{array}$ \\
\hline Produção de etileno & Atividade fixadora de $\mathrm{N}_{2}$ & mmol.planta ${ }^{-1} \cdot \mathrm{s}^{-1}$ \\
\hline Resistência & Estomatal & s.m $\mathrm{m}^{-1}$ \\
\hline Potencial & Osmótico, água & $\mathrm{J} . \mathrm{kg}^{-1}, \mathrm{~J} \cdot \mathrm{mol}^{-1}, \mathrm{kPa}$ \\
\hline Pressão & Osmótica, água & $\mathrm{Pa}$ \\
\hline Condutividade elétrica & Salinidade (solo, soluções) & $S \cdot m^{-1}, d S \cdot m^{-1}$ \\
\hline \multirow[t]{4}{*}{ Densidade de fluxo } & Fluxo de calor & W. $m^{-2}$ \\
\hline & Fluxo de água & kg. $m^{-2} \cdot s^{-1}, m^{3} \cdot m^{-2} \cdot s^{-1}, m^{-2} \cdot s^{-1}$ \\
\hline & Fluxo de fótons (fotossintese) & $\mu \mathrm{mol} . \mathrm{m}^{-2} \cdot \mathrm{s}^{-1}$ \\
\hline & Irradiância (fotossíntese) & W. $\mathrm{m}^{-2}$ \\
\hline Radioatividade & Materiais radioativos & $\mathrm{Bq}$ \\
\hline
\end{tabular}

Fonte: CANTARELLA e ANDRADE (1992).

2 Em alguns casos é aceitável a utilização de concentração em massa, por exemplo, concentração de micronutrientes em plantas.

QUADRO 9 - Fatores de conversão para unidades do Sistema Internacional (SI) de algumas unidades atualmente empregadas $^{(1)}$

\begin{tabular}{|c|c|c|c|}
\hline MEDIDA & UNIDADE FORA DO SI & MULTIPLICAR POR & UNIDADE SI \\
\hline \multirow[t]{2}{*}{ Comprimento } & Ângströn (-) & 0,1 & $\mathrm{~nm}$ \\
\hline & $\begin{array}{l}\text { mícron (:) } \\
\text { Caloria }\end{array}$ & $\begin{array}{l}1,0 \\
4,19\end{array}$ & $: \mathrm{m}$ \\
\hline \multirow{2}{*}{ Energia, calor } & BTU & 1.054 & $\mathrm{~J}$ \\
\hline & erg & $10^{-7}$ & $\mathrm{~J}$ \\
\hline \multirow[t]{2}{*}{ Pressão } & atmosfera & 0,1013 & $\mathrm{MPa}$ \\
\hline & & $\begin{array}{l}0,1 \\
6,9 \times 10^{3}\end{array}$ & $\begin{array}{l}\mathrm{MPa} \\
\mathrm{Pa}\end{array}$ \\
\hline Condutividade & m.mho.cm ${ }^{-1}$ & 0,1 & S. $\mathrm{m}^{-1}$ \\
\hline Força & dina & $10^{-5}$ & $\mathrm{~N}$ \\
\hline Radioatividade & curie & $3,7 \times 10^{10}$ & $\mathrm{~Bq}$ \\
\hline Irradiância & cal. $\mathrm{cm}^{-2} \cdot \mathrm{min}^{-1}$ & & W. $m^{-2}$ \\
\hline Ângulo plano & graus & $1,75 \times 10^{-2}$ & $\mathrm{rad}$ \\
\hline
\end{tabular}

1 Fonte: CANTARELLA e ANDRADE (1992)

\section{AGRADECIMENTOS}

O autor manifesta seu agradecimento ao Engenheiro Agrônomo, Dr. HEITOR CANTARELLA, Pesquisador Científico do Instituto Agronômico de Campinas (IAC) e ao Professor Dr. J. C. DE
ANDRADE, do Instituto de Química da Universidade Estadual de Campinas (UNICAMP), que autorizaram a utilização quase que literal dos quadros de artigo semelhante que elaboraram em 1992. 


\section{REFERÊNCIAS}

1. AMERICAN SOCIETY OF AGRONOMY - ASA. Publications handbook and stile manual. Madison: ASA, 1988. 92p.

2. CANTARELLA, H.; ANDRADE, J.C. O sistema internacional de unidades e a ciência do solo. Boletim Informativo, Campinas, v.17, n.3, p.91-102, 1992.

3. INSTITUTO NACIONAL DE METROLOGIA, NORMALIZAÇÃO E QUALIDADE INDUSTRIAL - INMETRO. Quadro geral de unidades de medida: resolução do CONMETRO n 12/1988. Duque de Caxias: INMETRO, 1989. 20p.

4. INSTITUTO NACIONAL DE PESOS E MEDIDAS - INPM. SI - Sistema Internacional de Unidades. Brasília: INPM, 1971. [Tradução autorizada pelo BIPM da publicação "Le Sistème Intenationale d'Unités"]

5. SALISBURY, F.B. Sistème internationale: the use of SI units in planta physiology. Journal of Planta Physiology, Rockville, v.39, p.1-7, 1991.

6. THIEN, S.J.; OSTER, J.D. The international system of unitis and its particular application to soil chemistry. Journal of Agronomic Education, Madison: v.10, p.62-70, 1981. 